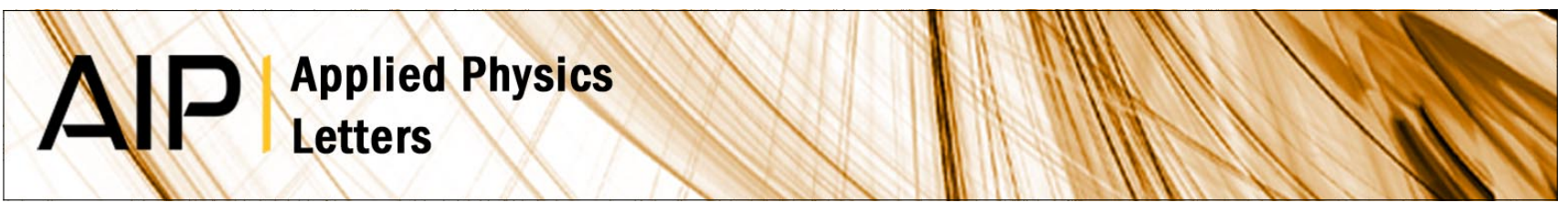

\title{
Optical activity and coupling in twisted dimer meta-atoms
}

Mingkai Liu, David A. Powell, llya V. Shadrivov, and Yuri S. Kivshar

Citation: Appl. Phys. Lett. 100, 111114 (2012); doi: 10.1063/1.3694269

View online: http://dx.doi.org/10.1063/1.3694269

View Table of Contents: http://apl.aip.org/resource/1/APPLAB/v100/i11

Published by the American Institute of Physics.

Additional information on Appl. Phys. Lett.

Journal Homepage: http://apl.aip.org/

Journal Information: http://apl.aip.org/about/about_the_journal

Top downloads: http://apl.aip.org/features/most_downloaded

Information for Authors: http://apl.aip.org/authors

\section{ADVERTISEMENT}
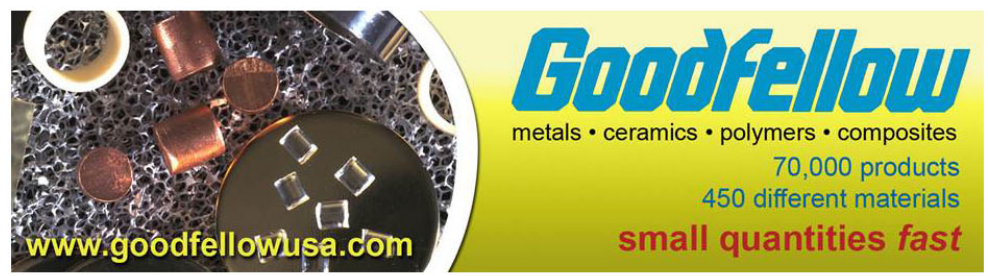


\title{
Optical activity and coupling in twisted dimer meta-atoms
}

\author{
Mingkai Liu, ${ }^{\text {a) }}$ David A. Powell, llya V. Shadrivov, and Yuri S. Kivshar \\ Nonlinear Physics Centre, Research School of Physics and Engineering, Australian National University, \\ Canberra, ACT 0200, Australia
}

(Received 10 December 2011; accepted 25 February 2012; published online 15 March 2012)

\begin{abstract}
We analyse the optical activity in twisted dimers, the meta-atoms of a chiral metamaterial, by introducing a simple yet accurate model for the coupling between them. The near-field interaction coefficients are derived from a Lagrangian model and include the effects of retardation, whereas the far-field radiation is based on a multipole expansion. We show that the optimum twist angle varies with frequency, and near resonance is substantially lower than 45 degrees, which is the lowest symmetry configuration. Our approach is accurate over a wide frequency range, including the resonant regions with the highest optical activity. In contrast to other models of near-field interaction, it requires no fitted parameters or homogenization procedure and is directly applicable to a wide variety of resonant particles. (C) 2012 American Institute of Physics.
\end{abstract}

[http://dx.doi.org/10.1063/1.3694269]

Subwavelength metallic structures can exhibit exotic electromagnetic properties at resonance. These resonant elements are widely studied as functional devices ${ }^{1,2}$ or as the building blocks of metamaterials. ${ }^{3,4}$ When two or more elements are assembled to form a hybrid structure, its resonant frequencies and far-field scattering properties can be tuned externally by modifying the spatial arrangement of elements. Recently, such hybrid structures have been extensively studied, including coupled wires, ${ }^{5}$ coupled split-ring resonators (SRRs), ${ }^{6}$ plasmonic oligomers, ${ }^{7}$ etc. Many interesting spectral features arise in these strongly coupled systems, including mode splitting, fano lineshape ${ }^{8}$ crossing or avoided crossing, ${ }^{9}$ and strong polarization rotation. ${ }^{10,11}$ Since the spectral features of these hybrid structures are strongly affected by the inter-particle separation, a deliberate study on the spatial dependence of the inter-particle interaction is required before experimental fabrication.

Twisted dimers are a particularly interesting class of coupled metamaterials, since their reduced symmetry makes them chiral, causing them to rotate the plane of polarisation of an incident wave. Examples include gammadions, ${ }^{10}$ crosses, ${ }^{11}$ and SRRs. ${ }^{12,13}$ The simplest of such structures is a pair of wires, which have been analytically studied in the low frequency limit. ${ }^{14}$ Such structures are also suitable for adding nonlinear inclusions, leading to strong nonlinear optical activity. ${ }^{15}$

In this letter, we study optical activity in a twisted wire dimer, showing that the resonant lineshapes can be easily predicted. This enables accurate modelling of the far-field scattering, clearly showing the polarisation rotation in all regimes, including at resonance where the effect is strongest. This is performed with a semi-analytical hybridisation model $^{6}$ of the coupled resonators, using coefficients derived from the numerically obtained charge and current distributions, ${ }^{16}$ and a multi-pole expansion of far-field radiation. In contrast to point-dipole based models ${ }^{17}$ our approach is ap-

\footnotetext{
${ }^{\text {a) }}$ Author to whom correspondence should be addressed. Electronic mail: lmk124@physics.anu.edu.au.
}

plicable to the close spacing typically used in twisted dimers.

The twisted cut-wire pair being studied is illustrated in Fig. 1. The first cut-wire (denoted as "1") is located on the plane $z=-s / 2$, oriented along the $y$ direction, and the second cut-wire (denoted as " 2 ") is on the plane $z=s / 2$, rotated about the $z$ axis by angle $\theta$. Our model starts from the Lagrangian description ${ }^{6}$ of the metallic structures, which provides an intuitive picture of the coupling and can predict the resonant frequencies as well as the polarization change in twisted SRRs. ${ }^{12}$ We utilise physically meaningful coupling parameters based on the calculated charge and current distribution. ${ }^{16}$ Including retardation effects in the calculation of the interaction constants allows the phase shift between coupled elements to be correctly predicted. ${ }^{18,19}$ We will show here that by including retardation effects in the self energy terms, both the near and far-field spectra can be quantitatively reproduced.

As described in Ref. 16, we numerically find the charge $\rho(t, \mathbf{r})$ and current distribution $\mathbf{J}(t, \mathbf{r})$ for a single wire and separate them into a time-dependent mode amplitude $Q(t)$ and spatially dependent charge density $q(\mathbf{r})$ and current

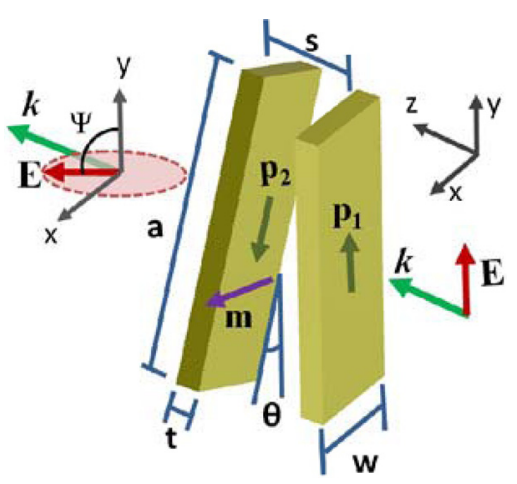

FIG. 1. (Color online) Schematic layout of the twisted cut-wire pair. The two cut-wires locate on the plane $z=-s / 2$ and $z=s / 2$, respectively, with a twist angle $\theta$ between them. We set their central points lying on the $z$ axis. The impinging wave propagates in the $z$ direction. 
density $\mathbf{j}(\mathbf{r})$. Following Ref. 12, the Lagrangian of the twisted cut-wire pair excited by a plane-wave is written as

$$
\begin{aligned}
\mathcal{L}= & \frac{L}{2}\left(\dot{Q}_{1}^{2}+\dot{Q}_{1}^{2}+2 \kappa_{M} \dot{Q}_{1} \dot{Q}_{2}\right)-\frac{1}{2 C}\left(Q_{1}^{2}+Q_{2}^{2}+2 \kappa_{E} Q_{1} Q_{2}\right) \\
& -\mathbf{p}_{1} \cdot \mathbf{E}_{i}-\mathbf{p}_{2} \cdot \mathbf{E}_{i} \exp (i \varphi) .
\end{aligned}
$$

where $Q_{m}, \dot{Q}_{m}$ describe the time-dependent amplitudes of the charge and current on wire $m(m=1$ and 2). $L$ and $C$ are the self-inductance and self-capacitance of a single cut-wire ${ }^{20}$ and $\kappa_{M}$ and $\kappa_{E}$ are magnetic and electric interaction coefficients, which are functions of $s$ and $\theta$. The last two terms of Eq. (1) describe the interaction energy between the cut-wire pair and the external field. $\mathbf{E}_{i}$ is the electric field of the incident wave, and $\varphi=\omega s / c$ is the phase difference in excitation of the wires due to retardation. $\mathbf{p}_{m}=Q_{m} \mathbf{l}_{\text {eff }}$ is the electric dipole moment of cut-wire $m$, where $l_{\text {eff }}=\left|\mathbf{l}_{\text {eff }}\right|$ $=\left|\int_{V_{m}} q(\mathbf{r}) \mathbf{r} \mathrm{d}^{3} \mathbf{r}\right|$ is the effective dipole length, which can be calculated by making a volume integral over cut-wire $m$ once $q(\mathbf{r})$ is known. According to the symmetry of the charge distribution, we can easily get $\mathbf{p}_{1}=\hat{\mathbf{y}} Q_{1} l_{\text {eff }}$ and $\mathbf{p}_{2}=Q_{2} l_{\text {eff }}(-\hat{\mathbf{x}} \sin \theta+\hat{\mathbf{y}} \cos \theta)$.

To obtain the solution for $Q_{m}$, we substitute Eq. (1) into the Euler-Lagrange equations

$$
\frac{d}{d t} \frac{\partial \mathcal{L}}{\partial \dot{Q}_{m}}-\frac{\partial \mathcal{L}}{\partial Q_{m}}=0, \quad(m=1 \text { and } 2) .
$$

Taking $\exp (-i \omega t)$ time dependence, the solution is

$$
\begin{gathered}
Q_{1}=\left(F_{\text {mut }} F_{2}-F_{\text {self }} F_{1}\right) /\left(F_{\text {self }}^{2}-F_{\text {mut }}^{2}\right), \\
Q_{2}=\left(F_{\text {mut }} F_{1}-F_{\text {self }} F_{2}\right) /\left(F_{\text {self }}^{2}-F_{\text {mut }}^{2}\right), \\
F_{\text {self }}=\left(\omega_{0}^{2}-\omega^{2}\right) \quad F_{\text {mut }}=\left(\omega_{0}^{2} \kappa_{E}-\omega^{2} \kappa_{M}\right), \\
F_{1}=\frac{l_{\text {eff }}}{L} E_{i, y} \quad F_{2}=\frac{l_{\text {eff }}}{L} e^{i \varphi}\left(E_{i, x} \sin \theta+E_{i, y} \cos \theta\right),
\end{gathered}
$$

$\omega_{0}=1 / \sqrt{L C}$, and $E_{i, x}$ and $E_{i, y}$ denote the $\mathrm{x}$ and y components of the incident electric field.

The coupling constants $\kappa_{E}$ and $\kappa_{M}$ can be determined by calculating the mutual electric and magnetic interaction energies including the retardation terms. ${ }^{18} \kappa_{E}=W_{E, 12} / W_{E, 11}$ and $\kappa_{M}=W_{M, 12} / W_{M, 11}$, where

$$
\begin{gathered}
W_{E, m n}=\int_{V_{m}} \int_{V_{n}} \frac{q^{*}(\mathbf{r}) q\left(\mathbf{r}^{\prime}\right) \exp \left(i k_{0}\left|\mathbf{r}-\mathbf{r}^{\prime}\right|\right)}{8 \pi \varepsilon_{0}\left|\mathbf{r}-\mathbf{r}^{\prime}\right|} \mathrm{d}^{3} \mathbf{r} \mathrm{d}^{3} \mathbf{r}^{\prime}, \\
W_{M, m n}=\int_{V_{m}} \int_{V_{n}} \frac{\mathbf{j}^{*}(\mathbf{r}) \cdot \mathbf{j}\left(\mathbf{r}^{\prime}\right) \exp \left(i k_{0}\left|\mathbf{r}-\mathbf{r}^{\prime}\right|\right)}{8 \pi \epsilon_{0} c^{2}\left|\mathbf{r}-\mathbf{r}^{\prime}\right|} \mathrm{d}^{3} \mathbf{r d}^{3} \mathbf{r}^{\prime},
\end{gathered}
$$

with $m, n \in\{1,2\}$ and $k_{0}=\omega / c$. For identical resonators, $W_{E, 11}=W_{E, 22}=1 / 2 C$ and $W_{M, 11}=W_{M, 22}=L / 2$. The retardation effect is taken into account in the calculation of $W_{E}$ and $W_{M}$ to get correct lineshapes. Now, it is clear that all the parameters needed to calculate $Q_{1}$ and $Q_{2}$ can be obtained deterministically from $q(\mathbf{r})$ and $\mathbf{j}(\mathbf{r})$.
Having determined the modal amplitudes $Q_{1}$ and $Q_{2}$, we use them to investigate the scattering properties. Undoubtedly, a rigorous calculation of the vector potential by making a volume integral of the current density can give us the field information from near-field to far-field region. But in the farfield zone, a multipole expansion of the twisted-dimer can already give us a good approximation. Here, we only take into account the three dominant terms: electric dipole p, electric quadrupole $\overline{\overline{\mathbf{q}}}$, and magnetic dipole $\mathbf{m}$. The radiated electric field at the far-field point $\mathbf{R}$ can be written explicitly as

$$
\begin{aligned}
\mathbf{E}(\mathbf{R})= & \frac{\exp \left(i k_{0} R\right)}{4 \pi \epsilon_{0} c^{2} R}\left\{\frac{-\omega^{2} \mathbf{R} \times(\mathbf{R} \times \mathbf{p})}{R^{2}}\right. \\
& \left.+\frac{i \omega^{3} \mathbf{R} \times[\mathbf{R} \times(\mathbf{R} \cdot \overline{\overline{\mathbf{q}}})]}{2 c R^{3}}-\frac{\omega^{2} \mathbf{R} \times \mathbf{m}}{c R}\right\} .
\end{aligned}
$$

From the symmetry of the charge and the current distribution, we can express the electric dipole, quadrupole, and magnetic dipole in terms of $Q_{m}, \theta$, and $l_{e f f}$

$$
\begin{aligned}
& \mathbf{p}=l_{\text {eff }}\left[-\hat{\mathbf{x}} Q_{2} \sin \theta+\hat{\mathbf{y}}\left(Q_{1}+Q_{2} \cos \theta\right)\right], \\
& \overline{\overline{\mathbf{q}}}=\frac{s l_{\text {eff }}}{2}\left[(\hat{\mathbf{y}} \hat{\mathbf{z}}+\hat{\mathbf{z}} \hat{\mathbf{y}})\left(Q_{2} \cos \theta-Q_{1}\right)-(\hat{\mathbf{x}} \hat{\mathbf{z}}+\hat{\mathbf{z}} \hat{\mathbf{x}}) Q_{2} \sin \theta\right], \\
& \mathbf{m}=\frac{i \omega s}{4} l_{\text {eff }}\left[\hat{\mathbf{x}}\left(Q_{2} \cos \theta-Q_{1}\right)+\hat{\mathbf{y}} Q_{2} \sin \theta\right] .
\end{aligned}
$$

By substituting these expressions into Eq. (7), we can readily calculate the far-field radiation patterns and analyse the contribution of each term.

To test our model, we compare the results of full wave simulation from CST Microwave Studio. In the calculation, we take a $=6 \mathrm{~mm}, t=0.1 \mathrm{~mm}, w=1 \mathrm{~mm}$, and $s=1 \mathrm{~mm}$. The metal is copper and the background is vacuum. The incident wave is polarized in the $y$ direction, parallel to cut-wire 1 . To probe the resonant spectral features, two near-field probes are positioned $0.1 \mathrm{~mm}$ away from the ends of the cut-wires; a far-field probe is used to detect the forward scattered wave in positive $\mathrm{z}$ direction.

Fig. 2 compares the results of $Q_{1}$ and $Q_{2}$ calculated using our model and the nearfield spectra from CST Microwave Studio (CST-MWS). Both the normalized
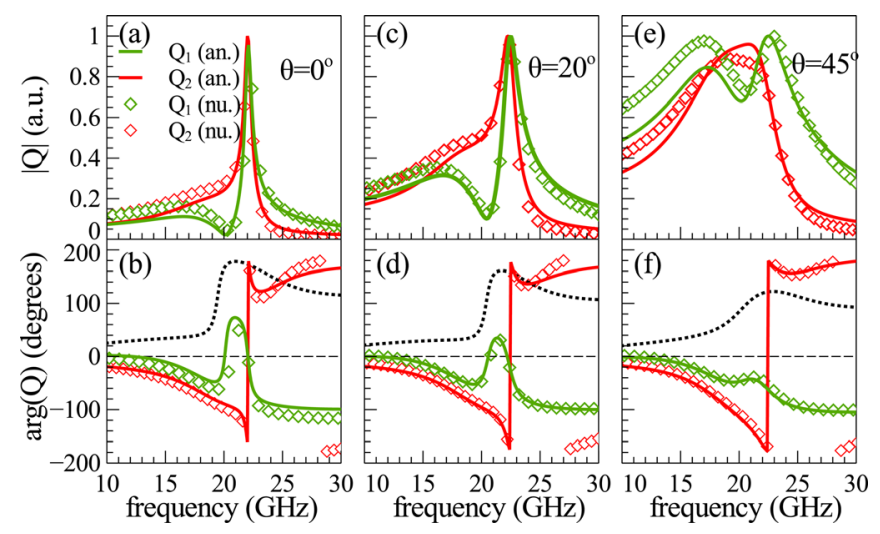

FIG. 2. (Color online) Normalized amplitudes and phases of $Q_{1}$ and $Q_{2}$ under different twist angle $\theta$. (a) and (b) $\theta=0^{\circ}$, (c) and (d) $\theta=20^{\circ}$, and (e) and (f) $\theta=45^{\circ}$. The results from our analytical model (an.) and numerical simulation (nu.) are plotted in (-) and $(\diamond)$, respectively. The black dotted line in the phase diagrams are the phase differences between $Q_{1}$ and $Q_{2}$ calculated with analytical model. 


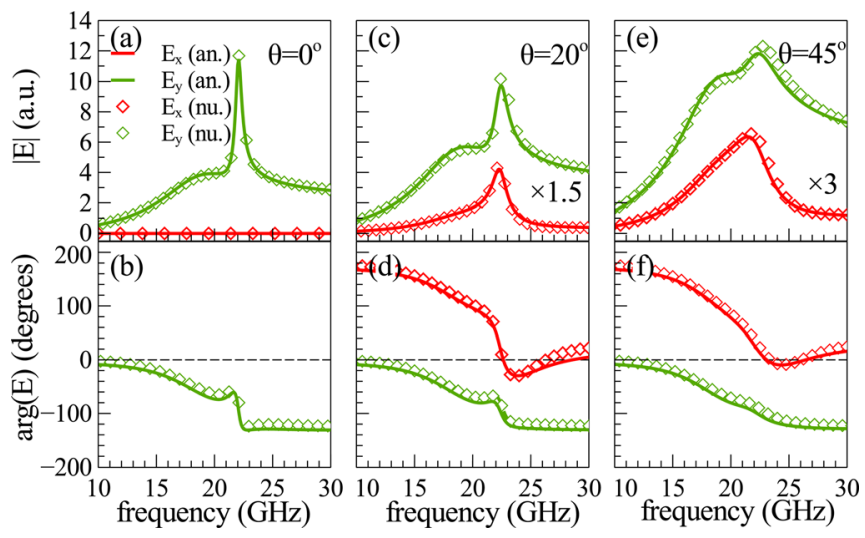

FIG. 3. (Color online) Amplitudes and phases of the electric field of forward radiated wave in positive $z$ direction, under different twist angles. (a) and (b) $\theta=0^{\circ}$, (c) and (d) $\theta=20^{\circ}$, (e) and (f) $\theta=45^{\circ}$. The results from analytical model (an.) and numerical simulation (nu.) are plotted in $(-)$ and $(\diamond)$, respectively.

amplitudes and the phases from the Lagrangian model have an overall good agreement with CST-MWS. Since the nearfield spectrum from a probe is position-dependent, it is inevitable that some differences show up. The sharp resonance at around $22 \mathrm{GHz}$ corresponds to the antisymmetric mode, as can be confirmed by the phase difference between $Q_{1}$ and $Q_{2}$ (black dotted line). The amplitude spectra show typical Fano lineshapes due to the interference between the broad superradiant symmetric mode and the narrow sub-radiant antisymmetric mode. We note that using only the real parts of the self and mutual energies correctly predicts the resonant frequencies, but gives erroneous lineshapes; therefore, we include all the imaginary terms. The correct physical interpretation of the "imaginary energies" is the power dissipated per period at the resonant frequency, primarily due to radiation losses.

Next, we compare the forward far-field radiation spectra, as shown in Fig. 3. The results have been normalized by the distance-dependent factor $\exp \left(i k_{0} z\right) / z$. When cut-wire 2 is twisted by an angle $\theta$, a cross component $E_{x}$ is observed in the radiated wave. For clarity, the electric field amplitudes of radiation are multiplied by 1.5 and 3 in Figs. 3(c) and 3(e), respectively. The excellent agreement between our method and numerical simulation is evident. Under a certain twist angle, the strongest cross polarization conversion occurs near the antisymmetric resonance, when $Q_{2}$ becomes maximum. This is consistent with Eq. (7); when $\mathbf{R}=(0,0, z)$, $E_{x}(\omega, R)=-\omega^{2} Q_{2} l_{\text {eff }} \sin \theta[1-i \omega s /(2 c)] \exp \left(i k_{0} z\right) /\left(4 \pi \epsilon_{0} c^{2} z\right)$.

The dependence of output polarization state on the twist angle and other parameters is of interest in the study of chiral structures. To show the efficiency of this model, we calculate the whole map of polarization states, when $\theta$ changes from $0^{\circ}$ to $90^{\circ}$ with a step of $1^{\circ}$, as shown in Fig. 4(a). It is found that the optimum twist angle varies with frequency, and the maximum polarization rotation occurs at around $\theta=40^{\circ}$, which is different from the result $\left(\theta=45^{\circ}\right)$ predicted from simple geometrical considerations. From the view point of symmetry, it is true that $45^{\circ}$ corresponds to the lowest symmetry. As has been predicted, ${ }^{14}$ for the transmission of a randomly oriented assembly, if the current spatial distribution and the interaction between neighbouring cut-wire pairs are

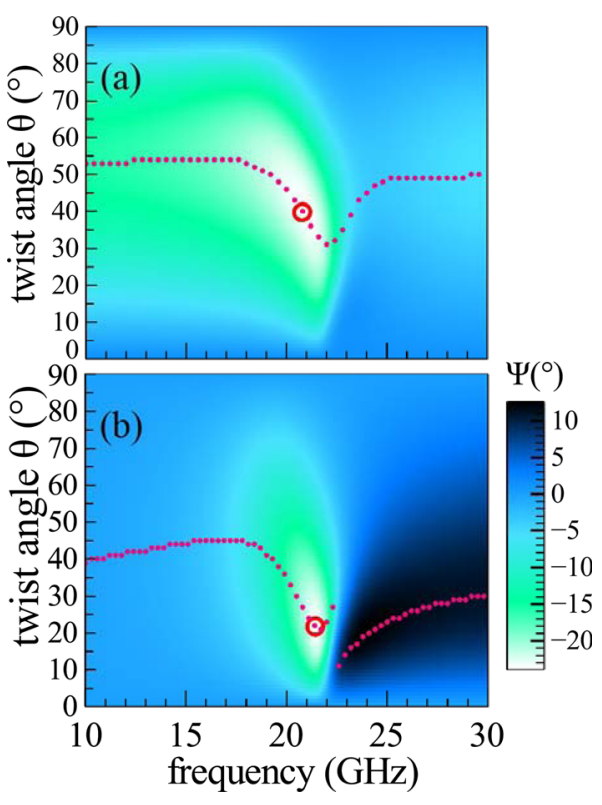

FIG. 4. (Color online) Polarization rotation angle $\Psi$ as a function of frequency and twist angle $\theta$. (a) For the scattered wave when cut-wire 1 is oriented along y direction; (b) for the averaged scattered wave when cut-wire 1 is oriented along $\mathrm{x}$ and $\mathrm{y}$ directions. The optimum twist angles for different frequencies are shown by the magenta dots, and the red circle denotes the position with maximum polarization rotation.

not taken into account, the optimum twist angle will locate at $45^{\circ}$ in the long-wavelength limit (far from resonance). This indicates that, in practical situation, only when the wires are infinitely thin and the structure is in the long-wavelength limit, the optimum angle can be $45^{\circ}$.

For the case studied here, such deviation can be attributed to several factors. The first is the intrinsic anisotropy of the cut-wire pair. The polarization rotation depicted in Fig. 4(a) is contributed by both the chirality and the anisotropy of the structure. To exclude the influence of anisotropy, we calculate the scattered light from the twisted cut-wire pair with its orientation rotated by $90^{\circ}$ (i.e., cut-wire 1 becomes oriented in $\mathrm{x}$ direction), and sum up with the original scattered light when cut-wire 1 is oriented in y direction. The polarization rotation angle of the averaged scattered light is shown in Fig. 4(b), and the optimum angle for each frequency is determined by the maximum absolute value of the polarization rotation angle. It can be seen that far from resonance, the polarization rotation angle is very small, since the effect of anisotropy is excluded. This result is consistent with previous predictions: ${ }^{14}$ if the two cut-wires are twisted about their central points, the polarization rotation effect of a randomly oriented assembly will disappear in the long-wavelength limit. However, the rotation effect remains strong near the antisymmetric mode, and the optimum angles are still strongly frequency dependent. Such behaviour is due to the second factor: strong phase fluctuation. The phase difference between the two resonators cannot be described approximately by $\exp \left(i k_{0}\left|\mathbf{r}-\mathbf{r}^{\prime}\right|\right)$ near the resonance, as shown in Fig. 2, this will perturb the conclusion in Ref. 14, and the optimum points change drastically. The third factor is the finite width of the wire. The current distribution in a finite-width cut-wire can no longer be considered as a line-current, and this can lead to different result for the optimum angle even 
in the long wavelength limit. The most extreme case is when $w=a$, in which case the angle of lowest symmetry becomes $22.5^{\circ}$. Thus, the lowest symmetry point for the case $w<a$ will be between 22.5 and $45^{\circ}$.

To conclude, we propose a semi-analytical method to study the resonant and scattering properties of chiral metallic structures based on a Lagrange model and multipole approximation. In this method, all the parameters can be obtained deterministically by considering the near-field interaction and the retardation effect. A twisted cut-wire pair is studied to test the model; both the resonant lineshapes and far-field results show a good agreement with full-wave simulation. This method can be further extended as an efficient tool for the future exploring on other chiral and hybrid structures. The approach can also be extended to include the coupling between neighbouring elements in an array. Council.

This work is supported by the Australian Research

${ }^{1}$ A. Ourir, A. de Lustrac, and J. M. Lourtioz, Appl. Phys. Lett. 88, 084103 (2006).

${ }^{2}$ J. Anker, W. Hall, O. Lyandres, N. Shah, J. Zhao, and R. Van Duyne, Nature Mater. 7, 442 (2008).

${ }^{3}$ D. R. Smith, J. B. Pendry, and M. C. K. Wiltshire, Science 305, 788 (2004).

${ }^{4}$ V. M. Shalaev, W. Cai, U. K. Chettiar, H. K. Yuan, A. K. Sarychev, V. P. Drachev, and A. V. Kildishev, Opt. Lett. 30, 3356 (2005).
${ }^{5}$ N. Liu, L. Langguth, T. Weiss, J. Kästel, M. Fleischhauer, T. Pfau, and H. Giessen, Nature Mater. 8, 758 (2009).

${ }^{6}$ H. Liu, Y. M. Liu, T. Li, S. M. Wang, S. N. Zhu, and X. Zhang, Phys. Status Solidi B 246, 1397 (2009).

${ }^{7}$ M. Hentschel, M. Saliba, R. Vogelgesang, H. Giessen, A. P. Alivisatos, and N. Liu, Nano Lett. 10, 2721 (2010).

${ }^{8}$ B. Luk'yanchuk, N. I. Zheludev, S. A. Maier, N. J. Halas, P. Nordlander, H. Giessen, and C. T. Chong, Nature Mater. 9, 707 (2010).

${ }^{9}$ A. Christ, S. Tikhodeev, N. Gippius, J. Kuhl, and H. Giessen, Phys. Rev. Lett. 91, 183901 (2003).

${ }^{10}$ A. V. Rogacheva, V. A. Fedotov, A. S. Schwanecke, and N. I. Zheludev, Phys. Rev. Lett. 97, 177401 (2006).

${ }^{11}$ M. Decker, R. Zhao, C. M. Soukoulis, S. Linden, and M. Wegener, Opt. Lett. 35, 1593 (2010)

${ }^{12}$ H. Liu, J. X. Cao, S. N. Zhu, N. Liu, R. Ameling, and H. Giessen, Phys. Rev. B 81, 241403 (2010).

${ }^{13}$ D. A. Powell, K. Hannam, I. V. Shadrivov, and Y. S. Kivshar, Phys. Rev. B 83, 235420 (2011).

${ }^{14}$ Y. Svirko, N. Zheludev, and M. Osipov, Appl. Phys. Lett. 78, 498 (2001).

${ }^{15}$ I. V. Shadrivov, V. A. Fedotov, D. A. Powell, Y. Kivshar, and N. I. Zheludev, New J. Phys. 13, 033025 (2011).

${ }^{16}$ D. A. Powell, M. Lapine, M. V. Gorkunov, I. V. Shadrivov, and Y. S. Kivshar, Phys. Rev. B 82, 155128 (2010).

${ }^{17}$ I. Sersic, C. Tuambilangana, T. Kampfrath, and A. Koenderink, Phys. Rev. B 83, 245102 (2011).

${ }^{18}$ A. Radkovskaya, E. Tatartschuk, F. Hesmer, C. Stevens, D. Edwards, and E. Shamonina, in 2nd International Congress on Advanced Electromagnetic Materials in Microwaves and Optics, Pamplona, Spain, 21-26 September 2008.

${ }^{19}$ L. Langguth and H. Giessen, Opt. Exp. 19, 22156 (2011).

${ }^{20}$ We interpret $L$ and $C$ as coefficients of stored energy, thus allowing their use despite the inapplicability of circuit theory. 